\title{
Intravital Laser-scanning Two-photon and Confocal Microscopy for Biomedical Research
}

Jieun Moon ${ }^{1,2}$

Pilhan $\mathrm{Kim}^{1,2,3}$

${ }^{1}$ Graduate School of Nanoscience and Technology,
Korea Advanced Institute of Science and
Technology (KAIST), Daejeon, Korea
${ }^{2}$ KI for Health Science and Technology, Korea
Advanced Institute of Science and Technology
(KAIST), Daejeon, Korea
${ }^{3}$ Graduate School of Medical Science and
Engineering, Korea Advanced Institute of Science
and Technology (KAIST), Daejeon, Korea

Received February 23, 2021

Accepted March 9, 2021

\section{Correspondence}

Pilhan Kim

Graduate School of Medical Science and

Engineering, Korea Advanced Institute of Science and Technology (KAIST), 291 Deahak-ro, Yuseonggu, Daejeon 34141, Korea

Tel.: +82-42-350-4865

Fax: +82-42-350-1110

E-mail: pilhan.kimवkaist.ac.kr

(C) Korean Society for Laser Medicine and Surgery

(c) This is an open access article distributed under the terms of the Creative Commons Attribution NonCommercial License (http://creativecommons.org/ licenses/by-nc/4.0) which permits unrestricted noncommercial use, distribution, and reproduction in any medium, provided the original work is properly cited.
Intravital microscopy is a high-resolution imaging technique based on laser-scanning two-photon and confocal microscopy, which allows dynamic 3D cellular-level imaging of various biological processes in a living animal in vivo. This unique capability allows biomedical researchers to directly verify a hypothesis in a natural in vivo microenvironment at the cellular level in a physiological setting. During the last decade, intravital microscopy has become an indispensable technique in several fields of biomedical sciences such as molecular and cell biology, immunology, neuroscience, developmental, and tumor biology. The most distinct advantage of intravital microscopy is its capability to provide a longitudinal view of disease progression at the cellular-level with repeated intravital imaging of a single animal over time by saving the images after each session.

\section{Key words}

Intravital microscopy; Two-photon microscopy; Confocal microscopy; In vivo 


\section{INTRODUCTION}

Intravital microscopy is a high-resolution imaging technique based on laser-scanning two-photon and confocal microscopy, which can enable a dynamic 3D cellular-level imaging of various biological processes in a living animal in vivo. This unique capability allows biomedical researchers to directly verify hypothesis in a natural in vivo microenvironment at cellular level in physiological setting. During the last decade, intravital microscopy has become an indispensable technique in wide area of biomedical science such as molecular and cell biology, immunology, neuroscience, developmental and tumor biology. ${ }^{1-3}$ It has been utilized to directly image gene expression, protein activity, drug delivery, cell trafficking, cell-cell interaction, and physiological response to external stimuli in a live animal. Real-time direct visualization of dynamic cellular processes in natural in vivo environment by intravital microscopy has provided unprecedented insights those were difficult to obtain by static ex vivo observation of thinly-sliced chemically-fixed tissues by conventional light microscopy (i.e. histological analysis). Although in vitro model studies have provided many significant findings with its advanced flexibility and manipulability over in vivo model studies, it has a fundamental limitation of failure to reconstitute the physiological microenvironment inside live tissue. Collectively, intravital microscopy based dynamic cellular imaging analysis in live animal model has become increasingly valuable in biomedical studies. Additionally, for drug development, intravital microscopy can be utilized as a unique tool for direct in vivo imaging analysis of new therapeutic candidates in various preclinical model of human diseases: imaging in vivo delivery of therapeutics to target tissues or cells for pharmacokinetics analysis, visualizing in vivo efficacy in the cell for pharmacodynamics analysis, and monitoring the projected mode of action (MOA) of therapeutics. Intravital imaging of drug candidates during in vivo experiment can not only accelerate the time-consuming optimization processes but also support fast decision making in the drug development. $^{4}$

\section{TECHNOLOGY FOR INTRAVITAL MICROSCOPY}

Utilizing a custom-built integrative intravital laser-scanning two-photon and confocal microscopy system, various internal organs and tissues of live mouse model can be visualized at cellular level by detecting fluorescence emitted from individual cells or microenvironment (Fig. 1). The major features of confocal and two-photon microscopy are summarized in Table 1. In both types of microscopy, image is created by observing fluorescence emitted
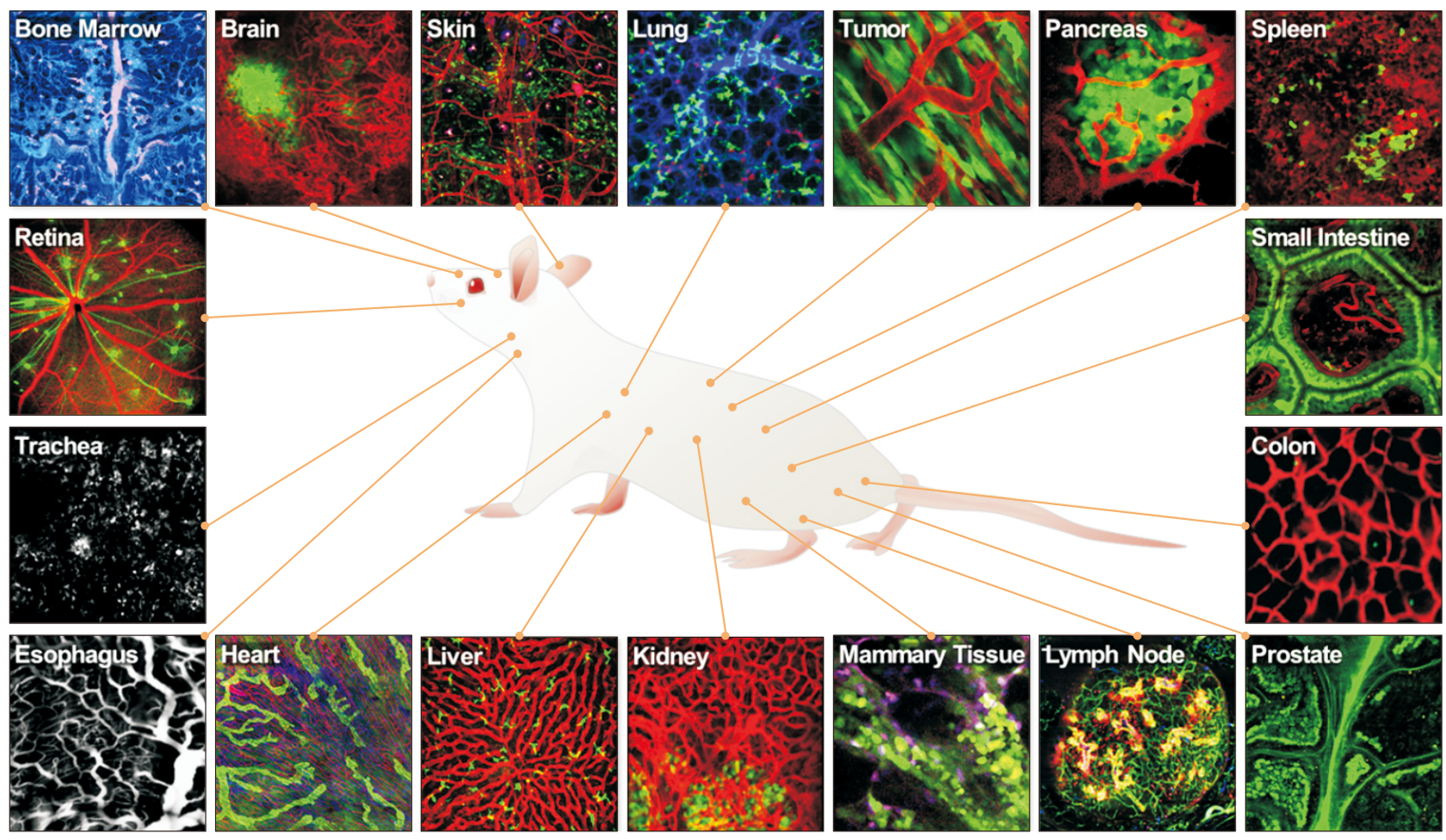

Fig. 1. Intravital cellular-level imaging of various organs in a live mouse model. 
Table 1. Comparison of confocal and two-photon laser-scanning microscopy

\begin{tabular}{lll}
\hline & Confocal laser-scanning microscopy & \multicolumn{1}{c}{ Two-photon laser-scanning microscopy } \\
\hline Sectioning principle & Pinhole rejecting out-of-focus fluorescence & Nonlinear fluorescence generation exclusively in focus \\
Laser type & Continuous wave laser & Femtosecond pulsed laser \\
Laser wavelength & $375-680 \mathrm{~nm}$ (typ.) & $700-1050 \mathrm{~nm}$ (typ.) \\
Excitation process & Single-photon absorption & Two-photon absorption \\
Pinhole before detector & Yes & No \\
De-scanning & Yes & No \\
\hline
\end{tabular}

from a fluorophore, which is categorized as fluorescence microscopy. In fluorescence microscopy, only cellularlevel objects of interests those are tagged by fluorophore are visible from an otherwise black background, thereby readily achieving high contrast and sensitivity even in in vivo condition. Additinoally, there are multiple advantages in fluorescence microscopy over other types of microscopy. First, over the past decades, tens of thousands fluorescent probes were synthesized by organic chemists to provide a highly versatile toolbox to label virtually any cellular components. Second, the development of fluorescent proteins, most notably green fluorescent protein (GFP), has allowed the intrinsic endogenous fluorescence labeling of various cells in live animal by common genetic manipulation, which alleviating difficulty in establishing in vivo labeling protocol using exogenous fluorescent probes. Notably, its profound impact on biomedical science is recognized by Novel Prize in Chemistry awarded at 2008 for the discovery and development of the GFP. Lastly, rapid technical advances in light sources and detectors optimized for fluorescence detection greatly aids fluorescence imaging to be an ideal modality allowing the detection of molecules and cells with superior signal-tobackground noise ratio over other imaging modalities.

In both types of microscopy, a tightly focused laser beam is scanned in two-dimensional Raster-pattern to achieve high-resolution optically sectioned imaging of live tissue. This tight focus is typically created by using high numerical aperture (NA) objective lens. The higher the NA of objective lens and the shorter the wavelength of light for excitation, the smaller the spot becomes to the diffraction-limit that is calculated to $0.61 \lambda / \mathrm{NA}$ in radius; $\lambda$ is the wavelength of the light. Although this diffractionlimited spot at the focal plane enables a highly localized fluorescence signal generation needed for a high-resolution imaging, still the excitation of fluorophore occurs non-selectively throughout the converging and diverging beam path at out-of-focal plane. Therefore, in confocal microscopy, a pinhole is placed in front of the detector at a position optically-conjugated to the focal plane and the pinhole aperture is aligned with the focus, which implements a highly effective rejection spatial filter to block the fluorescence from our-of-focus plane. It makes the fluorescence arise from the focus exclusively pass the pinhole aperture to the detector, thereby achieving optical sectioning.

On the other hand, two-photon microscopy achieves optical sectioning based on the nonlinear optical process, two-photon absorption. It is a phenomenon occurs when two seperate photons arrive at the same fluorophore at the same time within $\sim 0.5$ fs time difference and are absorbed together to combine their energy to excite the fluorophore. To achieve enough photon flux required for two-photon excitation, mode-locked Ti:Sapphire fs-pulse laser generating ultrashort pulse with 50-200 femtoseconds pulsewidth at about $80 \mathrm{MHz}$ repetition rate is commonly used for two-photon microscopy. In addition, the high photon density sufficient for two-photon absorption is achieved only at the very small volume at the focus. Thereby, the two-photon excited fluorescence is generated only from the focal plane, which facilitates intrinsic sectioning without additional components such as pinhole in confocal microscopy. De-scanning is the back-propagation of the fluorescence through the laser scanner optics. It is required only for confocal microscopy to implement stationary focus at the pinhole optically conjugated with $2 \mathrm{D}$ scanning beam inside the sample.

For the high NA objective lens, the working distance defined by the distance between the objective lens and the focus is typically in the range of several hundreds of micrometers to $1 \mathrm{~mm}$, thereby a surgical procedure is normally required to obtain a high-resolution image from internal visceral and thoracic organs. After the access to the organ and tissue surface, the confocal microscopy typically achieves non-invasive imaging depth up to 100 $150 \mu \mathrm{m}$ depending on type of tissue. Soft tissue normally provides more imaging depth than hard tissue. Bloodrich tissue has tendency to limit the imaging depth to less value than other tissue. In comparison, two-photon microscopy can achieve improved imaging depth in vari- 
ous tissue than the confocal microscopy, as it uses longer wavelength at near infrared (NIR) band from $700 \mathrm{~nm}$ to $1000 \mathrm{~nm}$ where the degree of light scattering limiting the imaging depth is greatly reduced than that in the visible wavelength band from $360 \mathrm{~nm}$ to $640 \mathrm{~nm}$ used in the confocal microscopy. Additionally, two-photon microscopy can detect second harmonic generation $(\mathrm{SHG})$ signals, which are optical signals generated when two photons of one wavelength are converted to a single photon of half the wavelength. As this process is purely optically-driven process, it does not require exogenous fluorescence labeling and enabled label-free imaging. Currently, SHG imaging is widely used to visualize collagen in the biological tissue.

\section{EXEMPLARY APPLICATION OF INTRAVITAL MICROSCOPY}

During last decade, intravital microscopic imaging of various internal organs including liver, ${ }^{5-7}$ spleen $^{8}$, pan-
A
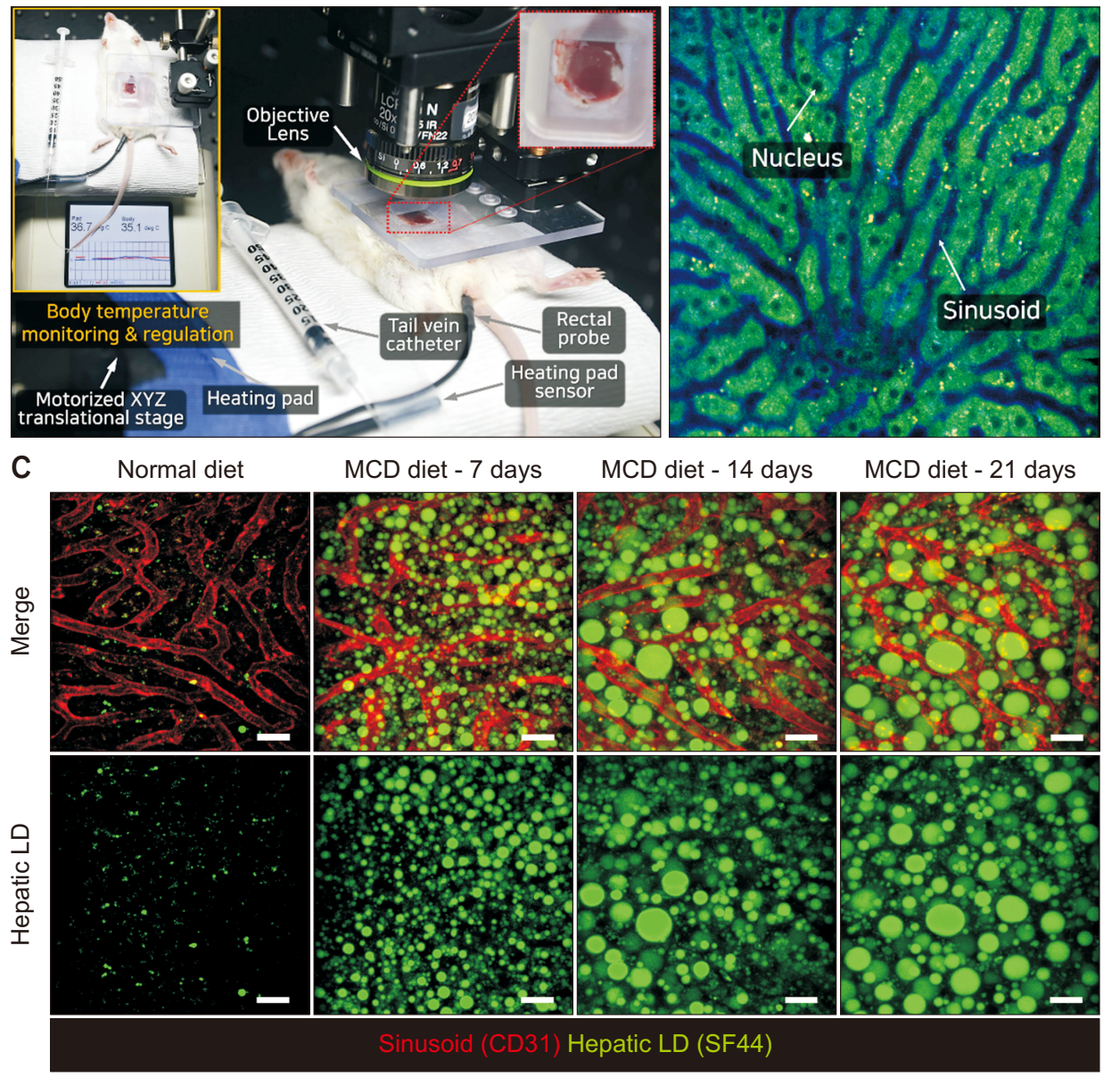

D

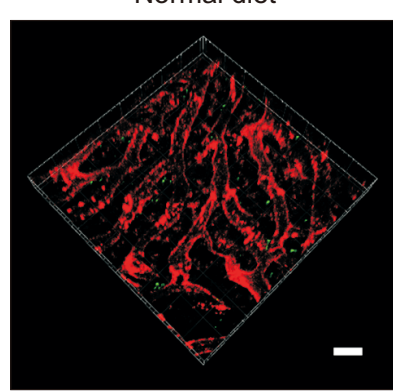

MCD diet - 7 days

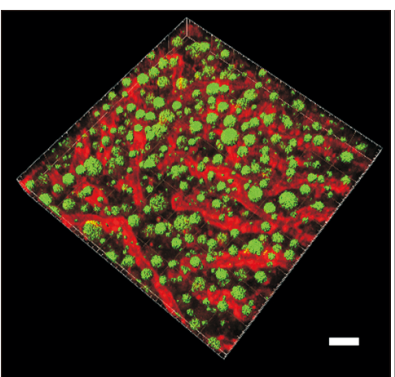

Hepatic LD (SF44)

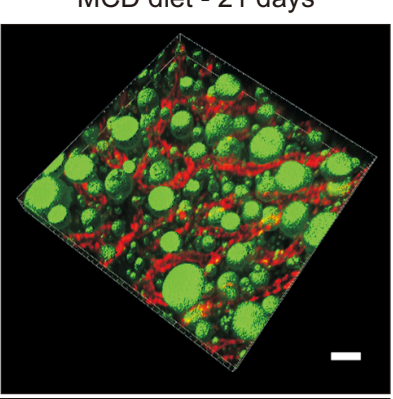

\section{B}

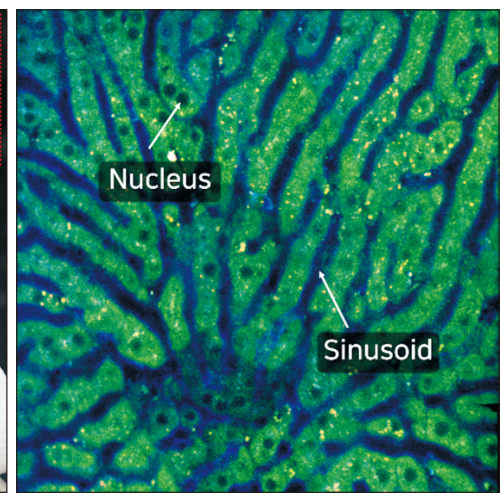

Fig. 2. Intravital imaging of liver of live mouse model. (A) Preparation of anesthetized mouse for intravital liver imaging. (B) Cellular-level imaging of liver showing nucleus of individual hepatocyte and hepatic sinusoid. (C, D) Longitudinal intravital imaging of liver showing hepatic lipid droplet accumulation in methionine and choline-deficient (MCD)-diet induced nonalcoholic fatty liver disease mouse model over 3 weeks. Maximal intensity projection image $(\mathrm{C})$ and 3-dimensionally rendered image (D) constructed from Z-stack images. Scale bars, 20 $\mu \mathrm{m}$. 
creas $^{9}$, kidney $^{10}$, small intestine, ${ }^{11,12}$ colon, ${ }^{13,14}$ lung, ${ }^{15,16}$ heart, ${ }^{17}$ prostate, ${ }^{18}$ mesentery ${ }^{19}$ and brain ${ }^{20}$ was achieved (Fig. 1). Fig. 2A shows the preparation of live mouse for the intravital imaging of liver. ${ }^{5-7}$ After anesthetization, small incision was made on skin and peritoneum to expose the left lobe of liver. The cover glass attached with a customized heater and commercial thermometer was positioned to gently press the exposed left lobe to reduce the liver movement. Additionally, a commercial homeothermic system composed of rectal probe for body temperature monitoring and feedback-controlled heating pad was used to maintain the body temperature of the anesthetized at $36^{\circ} \mathrm{C}$ during the intravital imaging. Fig. $2 \mathrm{~B}$ shows the microscopic-scale images obtained from the liver prepared for intravital imaging. Nucleus of individual hepatocyte and hepatic sinusoid was clearly visualized. The most distinct advantage of intravital microscopy is its capability of providing longitudinal view of disease progression in cellular-level with repeated intravital imaging of single animal over time by saving the animal after each imaging session. Fig. 2C, D shows the intravital imaging of the liver showing the progression of nonalcoholic hepatic steatosis in the nonalcoholic fatty liver disease (NAFLD) mouse model induced by feeding a methionine and choline-deficient (MCD) diet over 3 weeks. ${ }^{21,22}$ In each image session, liver sinusoid endothelial cells were labeled in vivo by intravenous injection of anti-CD31 monoclonal antibody conjugated with far-red fluorophore Alexa Fluor 647 at 2 hours before the intravital imaging. Additionally, hepatic lipid droplets (LDs) were labeled in vivo by intravenous injection of SF44 at 30 minutes before the intravital imaging. ${ }^{5,23}$ As clearly shown in Fig. $2 \mathrm{C}$, the size of the individual hepatic LDs in the hepatocytes gradually increased until 3 weeks with the continued feeding of the MCD diet. After 1 week of the MCD diet, the formation of hepatic LDs with both of the macrovesicular-shape and the microvesicular-shape was clearly observed. With progression of nonalcoholic hepatic steatosis, the number of hepatic LDs was rather decreased while the size of the individual LDs was significantly increased. Over time the ratio of macrovesicular-shaped hepatic LDs became larger than the microvesicular-shaped hepatic LDs. After 3 weeks of the MCD diet, many very large LDs with a diameter larger than $10 \mu \mathrm{m}$ were observed. In contrast, in the liver of the mouse fed the normal standard diet, only a few small hepatic LDs mostly smaller than $1 \mu \mathrm{m}$ in diameter were observed. Researches to elucidate an unknown pathophysiology in NAFLD has been mostly focused on investigating molecular or signaling pathways. Direct intravital imaging analysis of spatiotemporal alterations of hepatic microenvironment at cellular and subcellular levels during NAFLD progression as illustrated in Fig. 2 is a highly valuable method to pioneer new insightful research direction because conventional histological observation of postmortem liver tissue has many difficulties in dissecting the dynamically varying pathological features of NAFLD progression.

\section{CONCLUSION}

To conclude, intravital imaging and in vivo visualization approach have a unique advantage over conventional histology in that dynamic and temporal development of various human diseases in preclinical animal model can be analyzed in much more detailed manner at cellular level with rich molecular and functional information. Intravital microscopy will be a highly useful method for future investigation to explore unknown cellular and molecular mechanism in the pathogenesis of various human diseases.

\section{CONFLICT OF INTEREST}

No potential conflict of interest relevant to this article was reported.

\section{FUNDING}

None.

\section{REFERENCES}

1. Pittet MJ, Weissleder R. Intravital imaging. Cell 2011;147:98391.

2. Germain RN, Robey EA, Cahalan MD. A decade of imaging cellular motility and interaction dynamics in the immune system. Science 2012;336:1676-81.

3. Ellenbroek SI, van Rheenen J. Imaging hallmarks of cancer in living mice. Nat Rev Cancer 2014;14:406-18.

4. Conway JR, Carragher NO, Timpson P. Developments in preclinical cancer imaging: innovating the discovery of therapeutics. Nat Rev Cancer 2014;14:314-28.

5. Moon J, Kong E, Lee J, Jung J, Kim E, Park SB, et al. Intravital longitudinal imaging of hepatic lipid droplet accumulation in a murine model for nonalcoholic fatty liver disease. Biomed Opt Express 2020;11:5132-46.

6. Oh BJ, Jin SM, Hwang Y, Choi JM, Lee HS, Kim G, et al. Highly angiogenic, nonthrombogenic bone marrow mononuclear cellderived spheroids in intraportal islet transplantation. Diabetes 2018;67:473-85. 
7. Hwang Y, Yoon H, Choe K, Ahn J, Jung JH, Park JH, et al. In vivo cellular-level real-time pharmacokinetic imaging of free-form and liposomal indocyanine green in liver. Biomed Opt Express 2017;8:4706-16.

8. Choi H, Kim Y, Mirzaaghasi A, Heo J, Kim YN, Shin JH, et al. Exosome-based delivery of super-repressor IKB $\alpha$ relieves sepsis-associated organ damage and mortality. Sci Adv 2020;6:eaaz6980.

9. Park I, Hong S, Hwang Y, Kim P. A novel pancreatic imaging window for stabilized longitudinal in vivo observation of pancreatic islets in murine model. Diabetes Metab J 2020;44:193-8.

10. Lee EM, Park I, Lee YJ, You YH, Kim JW, Kim MJ, et al. Effect of resveratrol treatment on graft revascularization after islet transplantation in streptozotocin-induced diabetic mice. Islets 2018;10:25-39.

11. Choe K, Jang JY, Park I, Kim Y, Ahn S, Park DY, et al. Intravital imaging of intestinal lacteals unveils lipid drainage through contractility. J Clin Invest 2015;125:4042-52.

12. Ahn J, Choe K, Wang T, Hwang Y, Song E, Kim KH, et al. In vivo longitudinal cellular imaging of small intestine by side-view endomicroscopy. Biomed Opt Express 2015;6:3963-72.

13. Choi JW, Kim P, Kim JK, Kim YR, Fukumura D, Yun SH. Longitudinal tracing of spontaneous regression and anti-angiogenic response of individual microadenomas during colon tumorigenesis. Theranostics 2015;5:724-32.

14. Kim JK, Lee WM, Kim P, Choi M, Jung K, Kim S, et al. Fabrication and operation of GRIN probes for in vivo fluorescence cellular imaging of internal organs in small animals. Nat Protoc 2012;7:1456-69.

15. Park I, Kim M, Choe K, Song E, Seo H, Hwang Y, et al. Neutrophils disturb pulmonary microcirculation in sepsis-induced acute lung injury. Eur Respir J 2019;53:1800786.
16. Park I, Choe K, Seo H, Hwang Y, Song E, Ahn J, et al. Intravital imaging of a pulmonary endothelial surface layer in a murine sepsis model. Biomed Opt Express 2018;9:2383-93.

17. Jung K, Kim P, Leuschner F, Gorbatov R, Kim JK, Ueno T, et al. Endoscopic time-lapse imaging of immune cells in infarcted mouse hearts. Circ Res 2013;112:891-9.

18. Ghosh SK, Kim P, Zhang XA, Yun SH, Moore A, Lippard SJ, et al. A novel imaging approach for early detection of prostate cancer based on endogenous zinc sensing. Cancer Res 2010;70:611927.

19. Kim K, Choe K, Park I, Kim P, Park Y. Holographic intravital microscopy for 2-D and 3-D imaging intact circulating blood cells in microcapillaries of live mice. Sci Rep 2016;6:33084.

20. Lee J, Kong E, Hong S, Moon J, Kim P. In vivo longitudinal visualization of the brain neuroinflammatory response at the cellular level in LysM-GFP mice induced by 3-nitropropionic acid. Biomed Opt Express 2020;11:4835-47.

21. Anstee QM, Goldin RD. Mouse models in non-alcoholic fatty liver disease and steatohepatitis research. Int J Exp Pathol 2006;87:1-16.

22. Takahashi Y, Soejima Y, Fukusato T. Animal models of nonalcoholic fatty liver disease/nonalcoholic steatohepatitis. World J Gastroenterol 2012;18:2300-8.

23. Kim E, Lee S, Park SB. A Seoul-Fluor-based bioprobe for lipid droplets and its application in image-based high throughput screening. Chem Commun (Camb) 2012;48:2331-3.

How to cite this article: Moon J, Kim P. Intravital laserscanning two-photon and confocal microscopy for biomedical research. Med Lasers 2021;10:1-6. https://doi.org/10.25289/ ML.2021.10.1.1 